EESTI NSV TEADUSTE AKADEEMIA TOIMETISED. XII KOIDE

FOOSIKA-MATEMAATIKA-JA TEHNIKATEADUSTE SEERIA. 1963, NR. 4

ИЗВЕСТИЯ АКАДЕМИИ НАУК ЭСТОНСКОИ ССР. ТОМ ХІІ

СЕРИЯ ФНЗИКО-МАТЕМАТИЧЕСКИХ И ТЕХНИЧЕСКИХ НАУК. 1963, № 4

\title{
ОБ ИНТЕРПОЛЯТОРЕ, ОСУЩЕСТВЛЯЮЩЕМ ПЕРЕХОД НА ЭКВИДИСТАНТУ
}

\section{T. сиимут}

\section{Введение}

Прн обработке детали на фрезерном станке центр фрезы должен перемещаться по траекторнн, отстоящей от профиля детали на расстоянии, соответствующем радиусу прнменяемой фрезы, т. е. по эквидистанте. В настоящее время при цифровом програмнном управленни фрезерными станками исходная информация для интерполятора вычисляется для строго определенного радиуса фрезы, которой предусмотрено обработать данную деталь.

Поскольку даже небольшое отклонение радиуса используемой фрезы от запрограммированного вызывает изменения размеров детали, то возникли идеи компенсацин изменения радиуса фрезы в интерполяторе при его работе ['].

Ниже описывается принцип работы и структурная схема интерполятора, осуществляющего переход на эквидистанту на основе вводимых в него с программоносителя данных об обрабатываемом профиле и радиусе фрезы набираемого на пульте управления интерполятора.

\section{Принцип нахождения точки пересечения эквидистантных отрезков в интерполяторе}

Задачу любого интерполятора можно рассматривать как состоящую нз двух частей: 1) выдача закона движения центра фрезы между опорными точками; 2) обеспечение прекращения выдачи импульсов при достижении заданной опорной точки.

Ниже рассматривается принцип нахождения точки пересечения эквидистантных отрезков (опорной точки) в интерполяторе.

Заметим, что данный принцип применим в интерполяторах, воспронзводящих прямые линии и окружности, так как эквидистанты их являются соответственно прямой линией и окружностью.

Рассмотрим профиль $M N P$ (рис. 1), образованный пересечением отрезка окружности кр 1 с отрезком прямой пр 1, который обрабатывается фрезой радиусом $r_{\phi}$ при внутреннем обходе.

Уравнения окружности и прямой будут

$$
\begin{gathered}
\left(x-x_{0}\right)^{2}+\left(y-y_{0}\right)^{2}=R^{2} \\
y=k x+b
\end{gathered}
$$

$\left(x_{0}, y_{0}-\right.$ координаты центра окружности; $R$ - радиус окружности; $k$ - тангенс угла наклона прямой; $b$ - начальная ордината прямой). а уравнения эквндистант, соответственно, 


$$
\begin{gathered}
\left(x-x_{0}\right)^{2}+\left(y-y_{0}\right)^{2}=R_{3}^{2} \\
y=k x+b^{\prime} .
\end{gathered}
$$

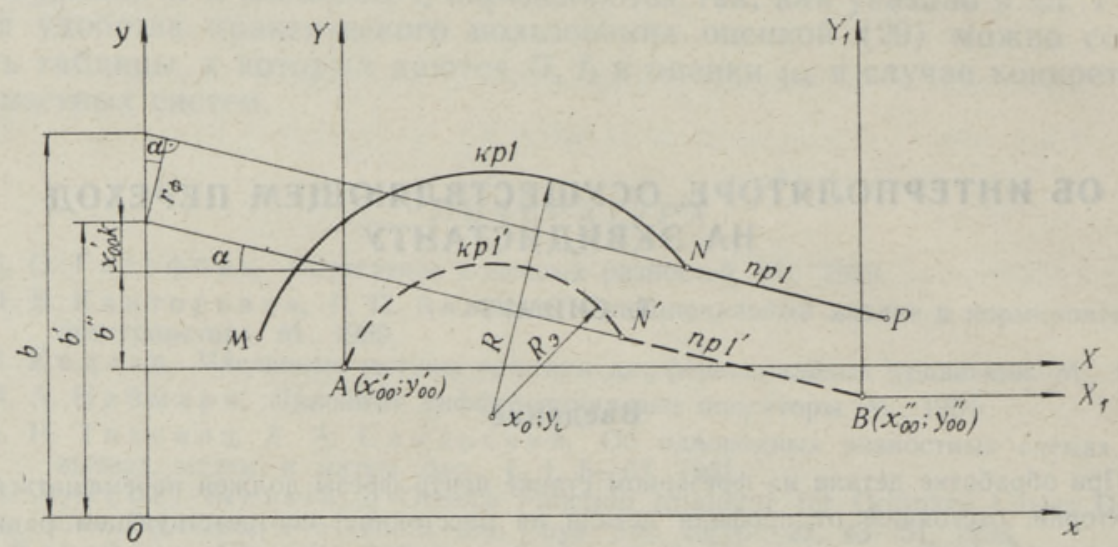

Рис. 1.

Нз рис. 1 видно, что

$$
\begin{gathered}
b^{\prime}=b-\frac{1}{\cos \alpha} r_{\phi} \\
R_{9}=R-r_{\phi} .
\end{gathered}
$$

Учтя (5) и (6), можно уравнения (3) и (4) записать в следующей форме:

$$
\begin{gathered}
x^{2}+y^{2}-2 x_{0} x-2 y_{0} y=R^{2}-x_{0}^{2}-y_{0}^{2}+r_{\phi}^{2}-2 R r_{\phi} \\
y-k x=b-\frac{1}{\cos \alpha} r_{\phi} .
\end{gathered}
$$

Поскольку (7) и (8) являются уравнениями эквидистант отрезков окружности кр 1 и прямой пр 1 , то точку пересечения этих эквидистантных отрезков можно найти решением системы уравнений (7) и (8). В принципе при пересечении прямой с окружностью или окружности с окружностью могут получиться две точки пересечения. Нужная точка пересечения должна определяться программой для интерполятора.

Движение центра фрезы в точку пересечения можно произвести двумя путями: а) фреза движется по окружности до пересечения с прямой; б) фреза движется по прямой до пересечения с окружностью.

Рассмотрим подробнее случай, когда центр фрезы движется по окружности кр $1^{\prime}$ до пересечения с прямой пр $1^{\prime}$.

Когда центр фрезы находится в точке пересечения кр 1' и пр 1', координаты этой точки $N^{\prime}$ должны удовлетворять условию (8). Для обнаружения такого положения центра фрезы необходимо сравнивать значения правой и левой частей уравнения (8).

Вычислим значения правой части уравнения (8) в единицах импульсов данной системы управления и введем его в регистр I (рис. 2). Левую часть уравнения (8) образуем в регистре II, где последовательно суммнруем отработанные интерполятором импульсы $y$ и произведенне $-k x$. При равенстве содержимых обоих регистров центр фрезы находится в точке пересечения кр 1' с пр 1'. 
Если начать движение центра фрезы из точки $A$ (рис. 1) и производить отсчет текущих координат от этой точки, можно образовать текущее содержимое регистра II простым суммированием импульсов ординаты и, при поступлении каждого импульса абсциссы, величины -kx. Но этим самым мы переносим начало координат из точки $(0 ; 0)$ в точку $\left(x_{00}^{\prime} ; y_{00}^{\prime}\right)-$ начальную точку траектории перемещения центра фрезы.

Уравнение (8) в новой системе координат $X, Y$ примет вид

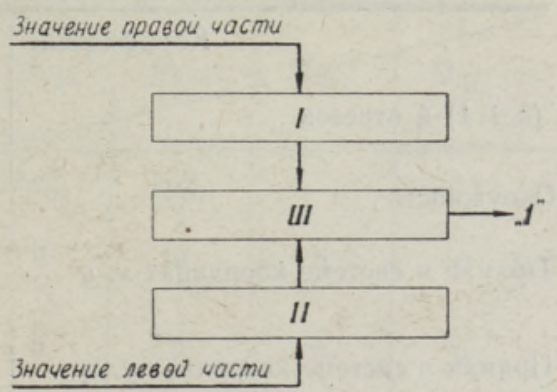

Рис. 2.

$$
Y-k X=b^{\prime \prime}
$$

причем

$$
b^{\prime \prime}=b-y_{00}^{\prime}-k x_{00}^{\prime}-\frac{1}{\cos \alpha} r_{\phi} .
$$

Таким образом, уравнение (9) будет

$$
Y-k X=b-k x_{00}^{\prime}-y_{00}^{\prime}-\frac{1}{\cos \alpha} r_{\phi} .
$$

Значит, в регистр I (рис. 2) необходимо ввести значение правой части уравнения (10) и сравнивать его с содержимым регистра II. При равенстве содержимого регистров I и II схема сравнения III выдает сигнал об окончании обработки данного отрезка профиля и для ввода новых данных в регистр I.

(Если $k>1$, то прямая рассматривается в системе координат $y, x$. При этом $x$ и $y$ в уравнении (10) меняют свои места.)

Если перемещение центра фрезы происходит от точки $\left(x_{00}^{\prime \prime} ; y_{00}^{\prime \prime}\right)$ по прямой пр 1' в направлении к окружности кр 1', то аналогичные вышеприведенным рассуждения приводят к условию, которому должны удовлетворять координаты точки пересечения траектории движения центра фрезы с окружностью кр 1', а именно:

$$
\begin{gathered}
X_{1}^{2}+Y_{1}^{2}-2 x_{0} X_{1}-2 y_{0} Y_{1}+2 x_{00}^{\prime \prime} X_{1}+2 y_{00}^{\prime \prime} Y_{1}= \\
=R^{2}-x_{0}^{2}-y_{0}^{2}+2 x_{0} x_{00}^{\prime \prime}+2 y_{0} y_{00}^{\prime \prime}-x_{00}^{\prime \prime}-y_{00}^{\prime 2}+r_{\phi}^{2}-2 R r_{\phi} .
\end{gathered}
$$

В общем случае условие, которому должны удовлетворять координаты точки пересечения эквидистантных отрезков обрабатываемого и последуюшего отрезков профиля, можно записать так:

$$
\begin{array}{r}
A_{n+1} X_{n}^{2}+B_{n+1} Y_{n}^{2}-C_{n+1} X_{n}-D_{n+1} Y_{n}+M_{n+1} X_{n}+N_{n+1} Y_{n}= \\
=E_{n+1}+C_{n+1} x_{00}^{n}+D_{n+1} y_{00}^{n}+P_{n+1} x_{00}^{n^{2}}+Q_{n+1} y_{00}^{n^{2}}+F_{n+1} r_{\phi}^{2}+G_{n+1} r_{\phi},
\end{array}
$$

где $X_{n}, Y_{n}$ - текущие координаты точек обрабатываемого отрезка в местной системе и $n$ - номер обрабатываемого отрезка, а $x_{10}^{n}, y_{00}^{n}-$ координаты начальной точки обрабатываемого отрезка в общей системе координат.

Значения коэффициентов приведены в табл. 1. 


\begin{tabular}{l|c|c|c|c|}
\hline Коэффициент & $A_{n+1}$ & $B_{n+1}$ & $C_{n+1}$ & $D_{n+1}$ \\
$(n+1)$-й отрезок & 1 & 1 & $2 x_{0(n+1)}$ & $2 y_{0(n+1)}$ \\
\hline Окружность & 0 & 0 & $k_{n+1}$ \\
Прямая в системе коордннат $x, y$ & 0 & 0 & -1
\end{tabular}

Знаки в уравнении (12) зависят как от знаков коэффициентов, так и от направления изменения текущих координат $X_{n}, Y_{n}$ обрабатываемого отрезка.

Для проверки выполнения условия (12) требуется устройство, которое во время обработки $n$-го отрезка вычисляет правую часть уравнения для $(n+2)$-го отрезка и одновременно сравнивает текущее значение левой части уравнения для $(n+1)$-го отрезка с заранее вычисленной его правой частью.

При равенстве обеих частей схема сравнения выдает сигнал «1» об окончании обработки $n$-го отрезка и для ввода новых коэффициентов (соответствующих отрезку с номером $n+2$ ).

\section{Структурная схема линейного интерполятора}

Как видно из уравнения (12) и табл. 1, техническое осуществление схемы линейного интерполятора будет намного проще, чем линейнокругового, так как большинство коэффициентов равно нулю. Ниже рассмотрим схему и принцип работы линейного интерполятора, осуществляюшего переход на эквидистанту.

Интерполятор состоит из двух основных блоков: 1) блок отработки и распределения импульсов по осям координат; 2) блок проверки выполнения условия (12).

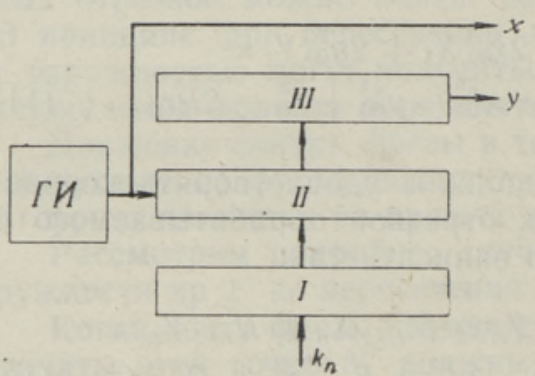

Рнс. 3.

Блок распределения импульсов (рис. 3) работает следующим образом. Перед началом работы интерполятора в регистр I вводится величина $k_{n}$ (тангенс угла наклона обрабатываемого отрезка). Во время работы импульсы поступают от генератора импульсов ГИ в привод оси $x$ и на вентили II. Импульс по оси $y$ выдается в случае переполнения регистра III.

Такая схема воспроизводит прямые c $\operatorname{tg} \alpha_{n}=k_{n} \leqslant 1$. Если $k_{n}>1$, то происходит переключение осей координат.

Уравнение (12) для линейного интерполятора таково:

$$
-C_{n+1} X_{n}-D_{n+1} Y_{n}=E_{n+1}+C_{n+1} x_{00}^{n}+D_{n+1} y_{00}^{n}+G_{n+1} r_{\phi} .
$$

Для проверки выполнения условия (13) необходимо ввести в интер- 
Таблица 1

\begin{tabular}{|c|c|c|c|c|c|c}
\hline$E_{n+1}$ & $F_{n+1}$ & $G_{n+1}$ & $M_{n+1}$ & $N_{n+1}$ & $P_{n+1}$ & $Q_{n+1}$ \\
\hline$R_{n+1}^{2}-x_{0(n+1)}^{2}-y_{0(n+1)}^{2}$ & 1 & $2 R_{n+1}$ & $2 x_{00}^{n}$ & $2 y_{00}^{n}$ & -1 & -1 \\
$b_{n+1}$ & 0 & $\frac{1}{\cos \alpha_{n+1}}$ & 0 & 0 & 0 & 0 \\
$b_{n+1}$ & 0 & $\frac{1}{\cos \alpha_{n+1}}$ & 0 & 0 & 0 & 0
\end{tabular}

полятор, помимо технологической информации, следующую информацию: 1) $\pm k_{n+1}$; 2) $\pm b_{n+1}$;3) $\pm \frac{1}{\cos a_{n+1}}$;4) условный знак, показывающий, в какой системе координат $(x, y$ или $y, x)$ рассматривается $(n+1)$-й отрезок профиля; 5) условный знак, показывающий увеличение или уменьшение текущей ординаты в соответственной системе координат ( $y$ в системе $x, y ; x$ в системе $y, x)$.

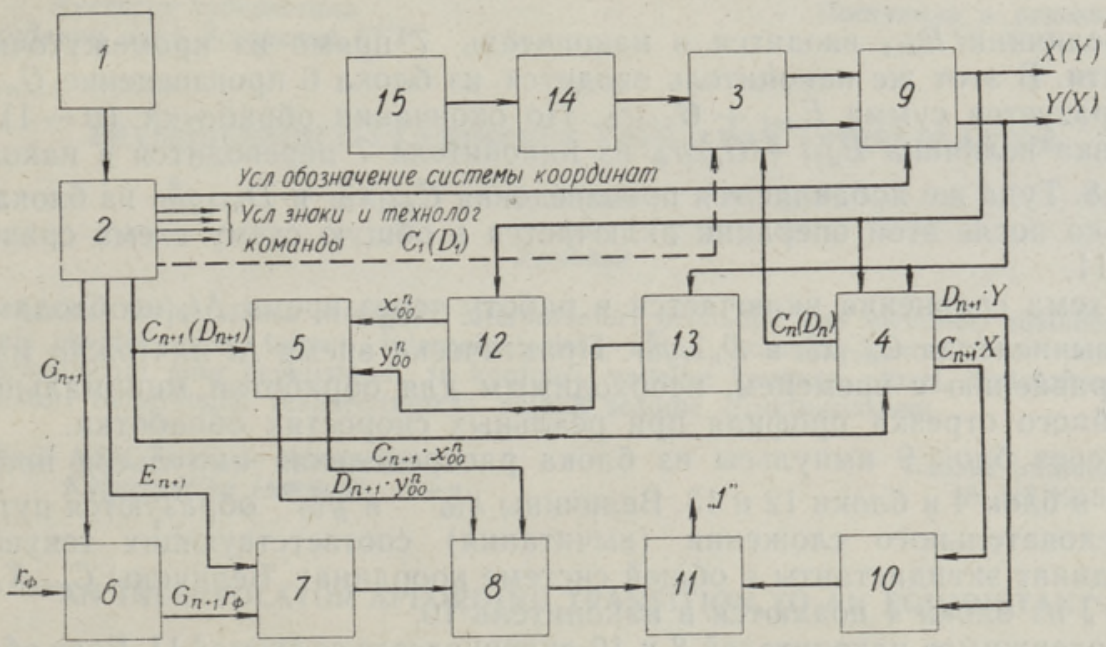

Рис. 4.

Блок-схема интерполятора изображена на рис. 4 , где

1 - устройство ввода, чтения и преобразования информации в двоичный код,

2 - промежуточная память интерполятора,

3 -- блок отработки и распределения импульсов по осям $x$ и $y$,

4 - блок получения величин $C_{n+1} X_{n}$ и $D_{n+1} Y_{n}$,

5 - блок образования произведений $C_{n+1} x_{00}^{n}$ и $D_{n+1} y_{00}^{n}$,

6 - блок образования произведения $G_{n+1} r_{\phi}$,

7 - накопитель с содержимым $E_{n+1}+G_{n+1} r_{\phi}$,

8 - накопитель с содержимым $E_{n+1}+C_{n+1} x_{00}^{n}+D_{n+1} y_{00}^{n}+G_{n+1} r_{\phi}$,

9 - блок перемены осей координат,

10 - накопитель с содержимым $C_{n+1} X_{n}+D_{n+1} Y_{n}$, 
11 - схема сравнения содержимого накопителей 8 и 10 ,

12 - блок образования $x_{00}^{n+1}$,

13 - блок образования $y_{00}^{n+1}$,

14 - генератор импульсов,

15 - блок регулирования скорости (частоты генератора импульсов)

Интерполятор работает следующим образом. Считываемая с перфоленты информация через устройство 1 вводится в промежуточную память 2. Из промежуточной памяти информация об условных знаках н технологические команды отпирают и запирают соответствующие вентили схемы логики интерполятора. Перед началом выдачи импульсов обработки $n$-го отрезка в блок 3 вводится $k_{n}\left(\right.$ т. е. $C_{n}$ или $\left.D_{n}\right)$.

При обработке первого отрезка $(n=1)$ величина $C_{1}\left(D_{1}\right)$ вводится в блок 3 прямо из промежуточной памяти. Для обработки последующих отрезков величина $C_{n}\left(D_{n}\right)$ берется из блока 4 , куда величина $C_{n}\left(D_{n}\right)$ была введена при обработке $(n-1)$-го отрезка. Коэффициент $C_{n+1}\left(D_{n+1}\right)$ вводится также в блок 5 . Коэффициент $G_{n+1}$ вводится в блок 6 . В этот же блок вводится набираемое на пульте значение $r_{\phi}$.

Блоки 3 и 4 работают в режиме интегратора с параллельным переносом, а блоки 5 и 6 есть обычные быстродействующие схемы умножения.

Величина $E_{n+1}$ вводится в накопитель 7 прямо из промежуточной памяти. В этот же накопитель вводится из блока 6 произведение $G_{n+1} r_{\phi}$ и образуется сумма $E_{n+1}+G_{n+1} r_{\phi}$. По окончании обработки $(n-1)$-го отрезка величина $E_{n+1}+G_{n+1} r_{\phi}$ из накопителя 7 переводится в накопитель 8. Туда же добавляются произведения $C_{n+1} x_{00}^{n}$ и $D_{n+1} y_{00}^{n}$ из блока 5 . Только после этой операции включается в общую схему схема сравнения 11 .

Схема сравнения включается в работу через время $\Delta t$, необходимое для вычисления $C_{n+1} x_{0 j}^{n}$ и $D_{n+1} y_{00}^{n}$. Практически время $\Delta t$ ничтожно мало по сравнению с временем, необходимым для обработки минимального линейного отрезка профиля при реальных скоростях обработки.

Через блок 9 импульсы из блока распределения импульсов поступают в блок 4 и блоки 12 и 13. Величины $x_{00}^{n+1}$ и $y_{00}^{n+1}$ образуются путем последовательного сложения (вычитания) соответствующих текущих координат эквидистанты в общей системе координат. Величины $C_{n+1} X_{n}$ и $D_{n+1} Y_{n}$ из блока 4 подаются в накопитель 10 .

Содержимое накопителей 8 и 10 сравнивается со схемой 11. Если абсолютная величина содержимого накопителя 10 окажется равной или больше содержимого накопителя 8 , то схема 11 срабатывает и выдает сигнал «1». После очистки блоков $3,4,5,6,7,8,10$ в них вводятся: в блок 3 из блока $4-C_{n+1}\left(D_{n+1}\right)$, а в блоки $4,5,6,7,8$ и 10 - информация о $(n+2)$-ом отрезке. Также переключается (если в этом есть надобность) схема логики интерполятора.

Тактовые импульсы получаем от генератора 14, частоту которых, а значит и скорость обработки, можно регулировать схемой 15 .

\section{Заключение}

1. Входная информация для интерполятора касается профиля детали, а не эквидистанты. Поэтому отпадает необходимость вычислять данные эквидистанты на УЦВМ, а вычисленная программа применима при обработке детали фрезой с любым радиусом, величина которого набирается перед началом обработки на пульте управления интерполятора. 
2. Все отрезки контура детали рассматриваются в единой системе координат, что устраняет возможность накопления, суммирования и переноса ошибок от одного отрезка профиля к другому (это показано сотрудником Института кибернетики АН ЭССР К. В. Леппиком).

3. Объем необходимой входной информации для рассмотренного линейного интерполятора остается малым.

4. Структура интерполятора несколько усложняется и увеличивается число используемых регистров. Длина регистров (количество триггерных ячеек) зависит, как и в существующих интерполяторах, от требуемой точности обработки и от длины максимального обрабатываемого линейного отрезка, а также от линейных размеров детали.

Автор выражает благодарность Ю. И. Пруудену за ценные замечания, сделанные им в ходе разработки данного интерполятора.

\section{ЛИТЕРАТ У РА}

1. К. В. Л е п п и к, О коррекции эквидистант при программном управленни фрезерными станками. Изв. АН ЭССР. Сер. физ.-матем. и техн. наук, 1963, 3.

Ннститут кибернетики

Академии наук Эстонской ССР
Поступила в редакцню

15. VI 1963

\section{EKVIDISTANDILE OLEMINEKUT VOIMALDAV INTERPOLAATOR}

\section{T. Siimut \\ Resümee}

Artiklis kirjeldatakse niisuguse interpolaatori printsiipi, kus üleminek mistahes nöutavale ekvidistandile toimub interpolaatorisse antud kontuuri kohta käiva numbrilise informatsiooni ning ekvidistandi ja kontuuri vahelise kauguse alusel. Esitatakse interpolaatori tööprintsiibi realiseerimiseks vajalik seadme struktuuriskeem.
Eesti NSV Teaduste Akadeemia
Küberneetika Instituut
Saabus toimetusse
15. VI 1963

\section{AN INTERPOLATOR AFFORDING TRANSITION TO AN EQUIDISTANT}

\section{T. Siimut \\ Summary}

This paper deals with the principle of a linear-circular numerical director which produces equidistant curves to the given contour, using the numerical information of the contour to be machined and the distance between the contour and the equidistant desired. A mathematical analysis of the problem discussed and a general layout of the device are presented.

Academy of Sciences of the Estonian S.S.R.,

Received Institute of Cybernetics

June 15th, 1963 